\title{
DIPOLE WITH COIL BASED ON HIGH TEMPERATURE SUPERCONDUCTOR
}

\author{
I.Bogdanov, S.Kozub, K.Myznikov, A.Seletskiy, P.Shcherbakov, P.Slabodchikov, V.Sytnik, \\ L.Tkachenko, V.Zubko \\ Institute for High Energy Physics, Protvino, Moscow region, Russia, 142280 \\ I.Akimov, A.Shikov
}

All-Russian Scientific-Research Institute of Inorganic Materials, Moscow, Russia, 123060

\section{Abstract}

The dipole magnet having a current carrying element, using a tape composite conductor on base of high temperature superconducting ceramics Bi2223 embedded in a silver matrix has been developed. The operating field of the dipole is $1 \mathrm{~T}$ at temperature of liquid nitrogen. The main characteristics of the used high temperature superconducting tape are displayed. The cross-section geometry optimization with goals to reduce field nonlinearities in aperture up to $10^{-4}$ and to minimize the value of magnetic field on the coil was carried out. The main results of the numerical simulations as well as of the properties of high temperature superconductors are presented.

\section{INTRODUCTION}

A great progress in manufacturing technique of high temperature superconductors (HTS) and improvement of their properties as well as their cost saving were achieved last years $[1,2]$. Now long-length multiple-strand conductors on base of Bi2223 and Bi2212 ceramics in a silver matrix are commercially available. They possess a high current-carrying capability, which reaches a current density substantially higher $10^{4} \mathrm{~A} / \mathrm{cm}^{2}$ at $77 \mathrm{~K}$ in a selffield [3]. As a result a practical application of HTS conductors is now in a good development. First of all they can find a use in an accelerator technique. Thus the resolve to produce current leads from HTS conductors for superconducting magnets was made in a collider under construction $\mathrm{LHC}$ with $14 \mathrm{TeV}$ energy $[4,5]$. It reduces essentially a load on a cryogenic system. The operational experience with HTS conductors showed their reliability, characteristics reproducibility and manufacturability. It allows one to take on a magnetic system creation on base of HTS conductors. By now all large accelerator centers of the world perform this sort of works.

Two ways of a use of HTS conductors for a magnet creation are now outlined. The first one is connected with a creation of hybrid designs, in which the main coils made either from $\mathrm{NbTi}$ or $\mathrm{Nb}_{3} \mathrm{Sn}$ conductors produce a primary field. An additional insertion is built from HTS conductors, which have a weak dependence of currentcarrying capability against magnetic induction in strong magnetic fields at temperature of liquid helium. The magnetic field values of $10 \div 15 \mathrm{~T}$ are tried to reach in these magnets $[6,7]$. Magnets, which use an iron yoke for creation of a uniform magnetic field up to $2 \mathrm{~T}$ by replacing of conventional conductors with HTS conductors working at temperature of liquid nitrogen, are developed in the second variant. The main task for further development of super-energetic accelerators is to reduce their costs per unit of energy. The estimations show that the second variant, in case of essential cost saving of HTS conductors at the mass production, can be more preferable from the viewpoint of price reduction of accelerator complex and its operation cost.

This article presents the results of development of a design of a dipole magnet for $1 \mathrm{~T}$ with an iron yoke. The tape composite HTS conductor from Bi2223 is used as a current-carrying element. The operating temperatures are $65 \div 77 \mathrm{~K}$. The magnet design is calculated for the maximal field up to $2 \mathrm{~T}$ in case of the further growth of the current-carrying capability of wire from HTS conductor.

\section{PROPERTIES OF HTS TAPE}

The composite superconducting tape consists of Bi2223 ceramic filaments placed in a silver matrix. It is fabricated by "powder in tube" method [2]. The crosssectional dimensions of the tape with 61 filaments are $3.2 \times 0.25 \mathrm{~mm}^{2}$. The fill factor of wire over superconducting ceramics is equal to $30 \%$. The critical current $\mathrm{I}_{\mathrm{c}}$ of the tape is equal to $20 \div 25 \mathrm{~A}$ in the self-field at $77 \mathrm{~K}$ (using criteria of $\mathrm{E}=1 \mu \mathrm{V} / \mathrm{cm})$. The temperature lowering to $65 \mathrm{~K}$ increases the critical current at least twice as much. The critical current has a strong dependence against field, especially versus normal component $B_{n}[8]$ (Fig.1).

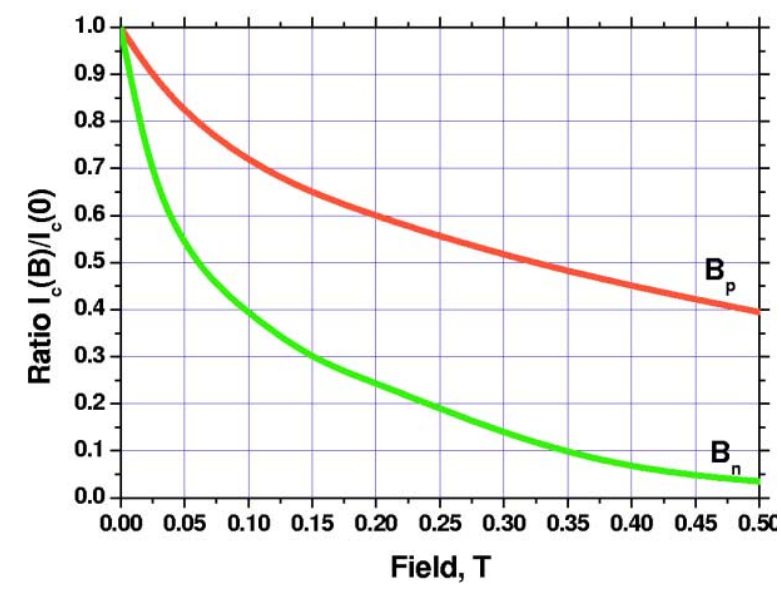

Fig.1. Dependence of critical current versus field at $77 \mathrm{~K}: \mathrm{B}_{\mathrm{n}}$ and $\mathrm{B}_{\mathrm{p}}$ are normal and parallel fields. 
Fig.2 illustrates the anisotropy of the critical currents for flat HTS conductors [9].

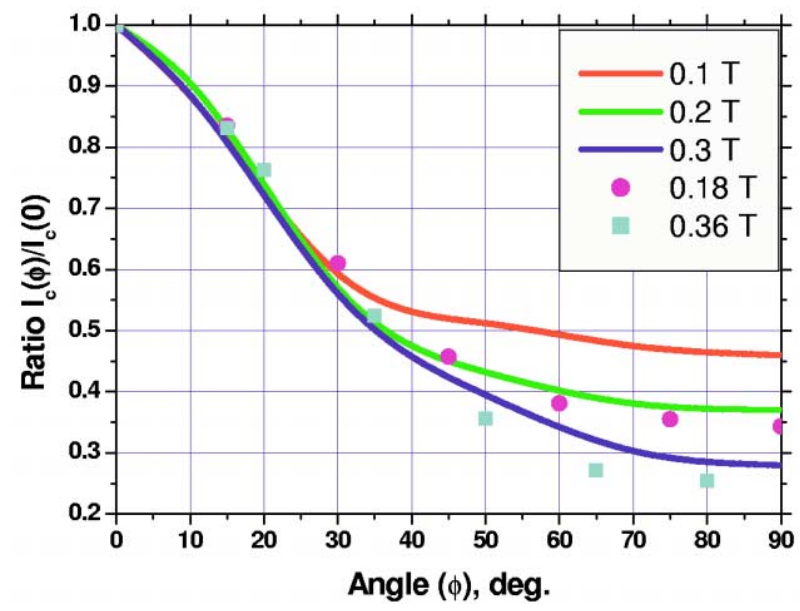

Fig.2. Field dependencies versus angle of field vector to surface of HTS tape. Solid lines are from the literature data; symbols are the experimental ones.

Also a degradation of the critical current versus pressure acting on a wide face of HTS tape was studied. The measurements showed the value of $I_{c}$ up to $5 \mathrm{MPa}$ is unaltered in the self-field and then a slow drop is observed up to $14 \mathrm{MPa}$ and the total fall is less then $5 \%$.

\section{OPTIMIZATION OF DIPOLE CROSS SECTION}

The first quadrant of the dipole cross section with labeled parameters for optimization is shown in Fig.3.

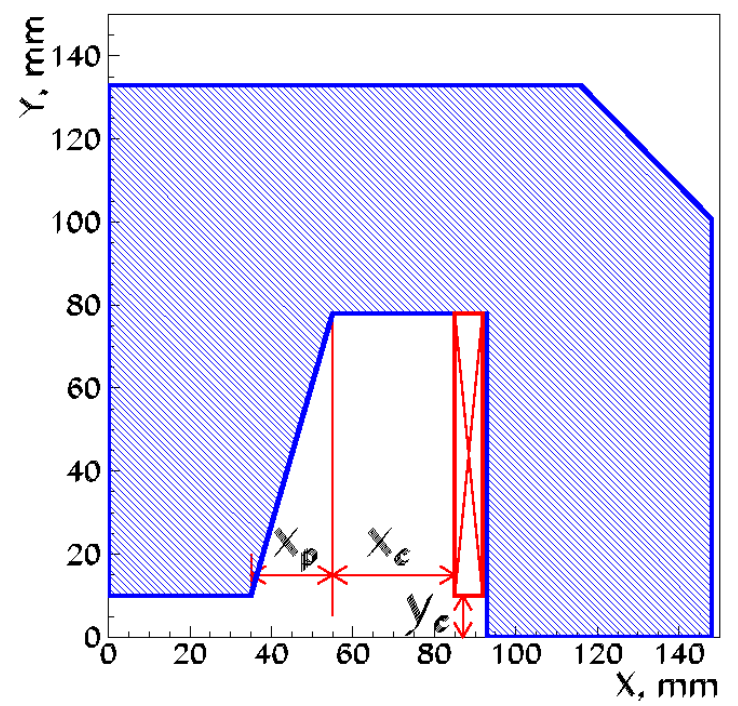

Fig.3. The general view of dipole cross-section.

Magnetic field simulation was carried out with help of the computer code MULTIC [10]. The steel quality of 2081 was accepted as a yoke material. It has wellmeasured magnetic characteristics both in small and large fields [11].

The magnetic field can be presented as a series:

$$
B_{y}+i B_{x}=B_{0} \sum_{n=1}^{\infty} W_{n}\left(\frac{r}{r_{0}} e^{i \Theta}\right)^{n-1},
$$

where $\mathrm{W}_{\mathrm{n}}=\mathrm{b}_{\mathrm{n}}+\mathrm{ia}_{\mathrm{n}}$ are the nonlinearities of $\mathrm{n}-1$ order, $\mathrm{B}_{0}$ is the central dipole field, $\mathrm{r}_{0}=10 \mathrm{~mm}$ is the reference radius. An ideal geometry has only odd nonzero nonlinearities, their influences on the beam dynamics are reduced against the growth of number $\mathrm{n}$, so only lower harmonics of order $n=3,5,7$ were considered during the process of optimization.

As a field homogeneity in aperture is formed only by iron poles, the dimensions of coil cross section $\left(6.8 \times 67 \mathrm{~mm}^{2}\right)$ have no influence on the field quality and were chosen only from the viewpoint of minimal HTS conductor consumption. Fig.4 shows the reduction of the lower nonlinearities with extension of the pole width. One can see the pole half-width must be not less than $35 \mathrm{~mm}$ in order to reach the values of the lower nonlinearities less than $10^{-4}$ at the pole gap of $20 \mathrm{~mm}$.

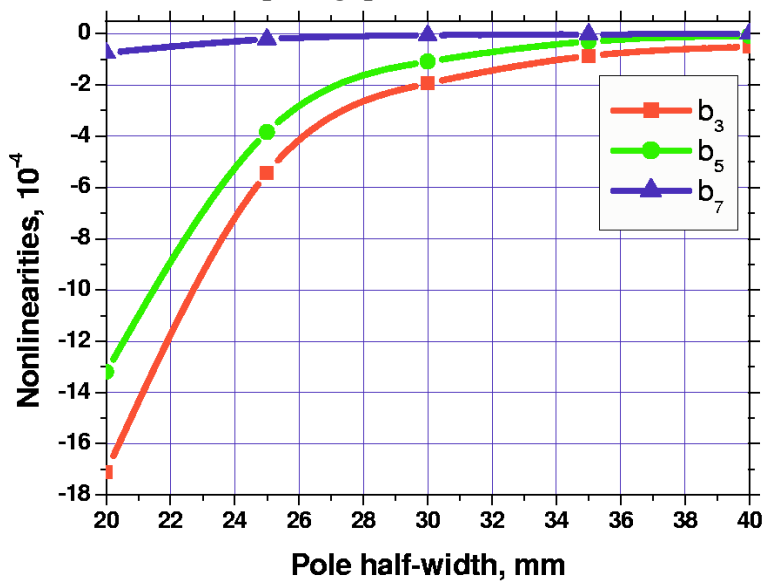

Fig.4. Dependencies of harmonic field components versus pole half-width.

One of the main goals for geometry optimization of the cross section is the minimization of the field in the coil that allows one to increase the critical current in the cable. Fig. 5 illustrates the influences of main geometric parameters on the values of field components in the coil. Parameter designations are clear from Fig.3. A winding of the coil was done with HTS conductor tape, the wide side of which had an orientation along Y-axis. As the critical current has the strong anisotropy in the magnetic field, $B_{x}$ component is the main factor for its determination. One can see from Fig. 3 a pole bevel (parameter $\mathrm{x}_{\mathrm{p}}$ ) as well as a coil moving off (parameter $\mathrm{x}_{\mathrm{c}}$ ) from the pole give a lowering of the magnetic field value in the coil. The coil separation from the median plane (parameter $y_{c}$ ) has a weak influence on the value of magnetic field. But this parameter allows one to use the coil shape like RACETRACK, which requires less HTS material in comparison with another shapes, for example as BEDSTEAD one. In that way it is possible to reduce the magnetic field down up to acceptable magnitude by the corresponding choice of the geometric parameters of $x_{p}, x_{c}$ and $y_{c}$. As a result the fol- 
lowing parameter points were chosen: $x_{p}=20 \mathrm{~mm}, x_{c}=$ $30 \mathrm{~mm}$ and $\mathrm{y}_{\mathrm{c}}=10 \mathrm{~mm}$. At the same time these parameters give also simultaneously the further reduction of the magnetic field nonlinearities of $b_{n}$ down to values less than $0.5 \times 10^{-4}$.

The coil cross-section has a strong elongated shape; the ratio of the coil height to the coil width is equal to approximately 10. An approaching of the cross-section shape to quadratic one with a conservation of the ampere turns and accordingly of the cross-section area results in a considerable growth of the magnetic field in the coil.

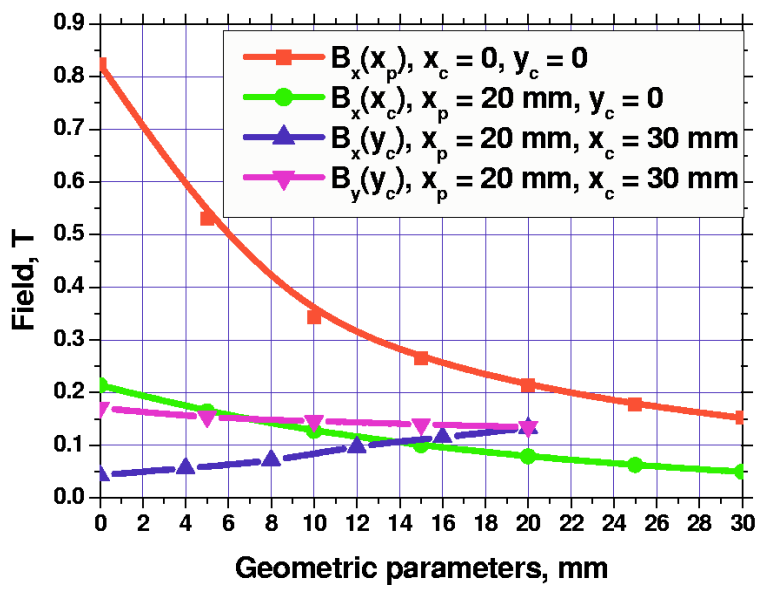

Fig.5. Influences of geometric parameters on field value in the coil.

The general view of the HTS dipole is shown in Fig.6 and the main parameters are found in Table 1.

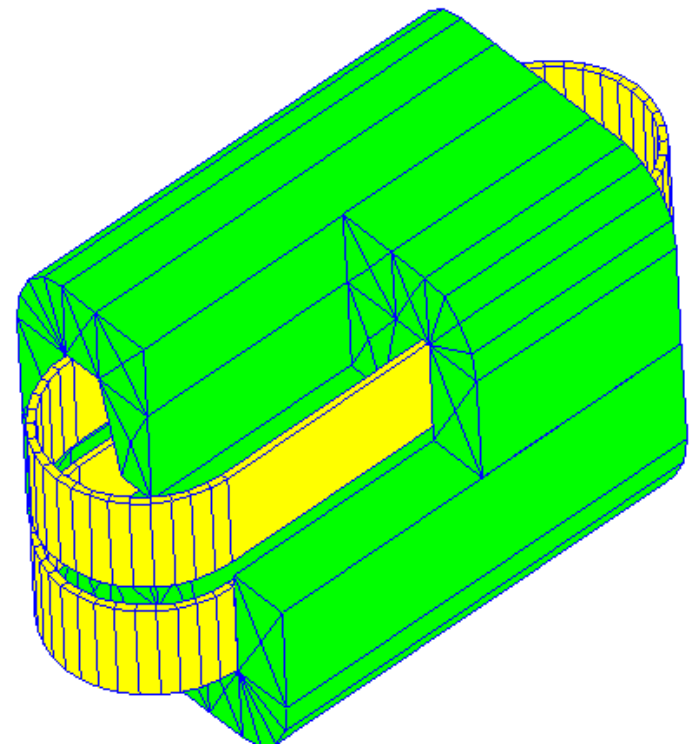

Fig.6. General view of HTS dipole.

\section{CONCLUSION}

The geometry optimization has been made in order to reduce the maximal field in the coil to acceptable level and to keep the high field quality in the aperture of the magnet. The minimization of the maximal field in the coil and high enough current-carrying capability of HTS conductors achieved at present time allow one to produce the dipole magnet with the nominal field of $1 \mathrm{~T}$ at $65 \div 77 \mathrm{~K}$, which can be increased up to $2 \mathrm{~T}$ under further improving of current-carrying capability.

Table 1. Main parameters of dipole.

\begin{tabular}{|l|c|}
\hline Nominal magnetic field, $\mathrm{T}$ & 1 \\
\hline Gap value between poles, $\mathrm{mm}$ & 20 \\
\hline Length of straight part, $\mathrm{mm}$ & 400 \\
\hline Total length, mm & 588 \\
\hline Pole width, $\mathrm{mm}$ & 70 \\
\hline Base width of pole, $\mathrm{mm}$ & 110 \\
\hline Height of coil, $\mathrm{mm}$ & 67 \\
\hline Width of coil, $\mathrm{mm}$ & 6.8 \\
\hline Gap between coils, mm & 20 \\
\hline Coil shift from pole base, $\mathrm{mm}$ & 30 \\
\hline Operating current, $\mathrm{A}$ & 18 \\
\hline Number of turns in coil & 450 \\
\hline Operating temperature, $\mathrm{K}$ & $65 \div 77$ \\
\hline Maximal field in coil, $\mathrm{B}_{\mathrm{x}}, \mathrm{T}$ & 0.08 \\
\hline Maximal field in coil, $\mathrm{B}_{\mathrm{y}}, \mathrm{T}$ & 0.15 \\
\hline
\end{tabular}

\section{REFERENCES}

[1]. D.C. Larbalestier and P.J.Lee, Prospects for the Use of High Temperature Superconductors in High Field Accelerator Magnets, Proc. of PAC'99, New York, 1999, p.177.

[2], A. Nikulin, A. Shikov, A. Akimov, HTS: from Investigation to Application, XVI Workshop on Charged Particle Accelerators, Protvino, 1999, v.2, p.13.

[3]. L. Mazur et al., Long Length Manufacturing of BSCCO-2223 Wire for Motor and Cable Applications. Int. Cryogenic Materials Conf., Montreal, Quebec, Canada, 1999.

[4]. A. Ballarino, Application of High Temperature Superconductors to Accelerators, Proc. of EPAC-2000, Vienna, Austria, 2000, p.227.

[5]. I. Bogdanov et al., Application of HTS Bi2223 for Current Leads of Superconducting Magnets, Proc. of EPAC-2000, Vienna, Austria, 2000, p.2178.

[6]. R. Gupta, Common Coil Magnet System for VLHC, Proc. of PAC'99, New York, 1999, p.3239.

[7]. A.K. Gosh et al., A Common Coil Magnet for Testing High Field Superconductors, Proc. of PAC'99, New York, 1999, p.3230.

[8]. A.D. Nikulin. Material Science Aspects of HTS Technical Superconductors. Physics and Material Science of High Temperature Superconductors, IV NATO ASI Series, 3. High Technology, 1997, V.26, p.129.

[9] P.E. Richens et al., Progress in Iron Cored High-Tc Magnet Development for Electromagnetic Actuator Applications, Proc. of MT-15, Beijing, China, 1997, p.785.

[10] L. Tkachenko, Code Package MULTIC for Calculation of Magnetic Field with an Arbitrary Configuration. IHEP Preprint 98-29, Protvino, 1998.

[11] A. Baydakov et al., Study of Magnetic Properties of Stainless and Electrical Steels at Low Temperatures for UNK Superconducting Dipoles. IHEP Preprint 92-77, Protvino, 1992. 